\title{
ON THE EXISTENCE OF GLOBAL SOLUTIONS OF THE NONLINEAR PARABOLIC EQUATION OF EELLS-SAMPSON OVER PRODUCT MANIFOLDS
}

\author{
SEIKI NISHIKAWA ${ }^{1}$
}

\begin{abstract}
We extend results of Lemaire and Hamilton on the existence of global solutions of the equation in the title to warped product manifolds with boundaries.
\end{abstract}

1. Introduction. The object of this note is to prove the following

THeOREM 1. Let $M=N \times_{\varphi} K$ and $M^{\prime}=N^{\prime} \times{ }_{\psi} K^{\prime}$ be warped products of compact Riemannian manifolds; $N, N^{\prime}$ being with boundary and $K, K^{\prime}$ without boundary. Suppose $N^{\prime}$ has nompositive sectional curvature everywhere and convex boundary $\partial N^{\prime}$. Let $F: K \rightarrow K^{\prime}$ be a harmonic map with constant energy density and $f_{0}: N \rightarrow N^{\prime}$ be a $C^{\infty}$ map. Let $u: \partial M \times[0, \infty) \rightarrow M^{\prime}$ be a $C^{\infty}$ map independent of time $t \in[0, \infty)$ and suppose $u=f_{0} \times F$ on the corner $\partial M \times 0$. Then the Dirichlet initial-boundary value problem for the nonlinear parabolic equation of Eells-Sampson

$$
\begin{aligned}
\partial U / \partial t & =\tau(U) \text { on } M \times[0, \infty), \\
U & =f_{0} \times F \quad \text { on } M \times 0, \\
U & =u \text { on } \partial M \times[0, \infty)
\end{aligned}
$$

has a global solution $U: M \times[0, \infty) \rightarrow M^{\prime}$, which is unique, and $C^{\infty}$ except on the corner $\partial M \times 0$.

For the terminology and the notation in the theorem, see $\$ 2$.

In [3], Hamilton proved that if $\boldsymbol{M}^{\prime}$ has nonpositive sectional curvature everywhere, then the Dirichlet problem (D) has a global solution $U(x, t)=U_{t}(x)$ : $M \times[0, \infty) \rightarrow M^{\prime}$ for arbitrary initial condition $U_{0}: M \times 0 \rightarrow M^{\prime}$ a $C^{\infty}$ map with $U_{0}=u$ on $\partial M \times 0$, and $\left\{U_{t}\right\}_{t}$ converges uniformly to a harmonic map $U_{\infty}$ : $M \rightarrow M^{\prime}, \tau\left(U_{\infty}\right)=0$ as $t \rightarrow \infty$.

In contrast with this, Theorem 1 shows that for a special class of initial conditions the Dirichlet problem (D) has a global solution even when the target manifold $M^{\prime}$ admits positive sectional curvature, for the warped product $M^{\prime}=N^{\prime}$ $\times_{\psi} K^{\prime}$ has positive sectional curvature by a suitable choice of scale factor $\psi$. It is, however, not known in general whether the solution $\left\{U_{t}\right\}_{t}$ subconverges to a harmonic map.

Received by the editors April 16, 1980.

1980 Mathematics Subject Classification. Primary 35K55; Secondary 58E20.

Key words and phrases. Nonlinear parabolic equation of Eells-Sampson, harmonic map, warped product.

${ }^{1}$ Partially supported by the Sakkokai Foundation. 
In the case where $N$ and $N^{\prime}$ are without boundary, Theorem 1 was proved by Lemaire [4]. I wish to thank him for suggesting this investigation and helpful discussion.

2. Preliminaries. Let $M=(M, g)$ and $M^{\prime}=\left(M^{\prime}, g^{\prime}\right)$ be compact Riemannian manifolds and $U: M \rightarrow M^{\prime}$ a $C^{\infty}$ map. Then the energy density $e(U)$ of $U$ is defined by $e(U)=\frac{1}{2}|d U|^{2},|d U|$ being the norm of the differential $d U$ of $U$, and the energy $E(U)$ of $U$ is defined by the integral

$$
E(U)=\int_{M} e(U) d \mu_{M}
$$

$U$ is called harmonic if it satisfies the Euler-Lagrange equation $\tau(U)=0$, which is a semilinear elliptic system of second order, of the energy functional $E$. The EulerLagrange operator $\tau(U)=\operatorname{Div}(d U)$ defines a $C^{\infty}$ vector field, called the tension field of $U$, on $M^{\prime}$ along $U$ and is expressed in local coordinates $\left(x^{i}\right),\left(y^{\alpha}\right)$ of $M$ and $M^{\prime}$ as

$$
\tau(U)^{\alpha}=\Delta U^{\alpha}+g^{i j}{ }_{M} \Gamma_{\beta \gamma}^{\alpha}(U) U_{i}^{\beta} U_{j}^{\gamma},
$$

where $\Delta$ is the Laplacian on $M,{ }_{M^{\prime}} \Gamma_{\beta \gamma}^{\alpha}$ are Christoffel symbols on $M^{\prime}$ and $U_{i}^{\alpha}=$ $\partial U^{\alpha} / \partial x^{i}$. See Eells-Sampson [2] for details.

Let $N=(N, g)$ and $K=(K, h)$ be Riemannian manifolds and $\varphi$ a positive $C^{\infty}$ function on $N$. Then their warped product $N \times{ }_{\varphi} K$ is defined to be the Riemannian manifold $(N \times K, g \times \varphi h), g \times \varphi h$ being the product metric of $g$ and $\varphi h$. For the fundamental properties of warped product manifolds, see Bishop-O'Neill [1].

For a Riemannian manifold $N$ with boundary $\partial N$, we say that $\partial N$ is convex if the second fundamental form of $\partial N$ in $N$ with respect to the inward unit normal vector is positive semidefinite everywhere.

For $C^{\infty}$ maps $f: N \rightarrow N^{\prime}$ and $F: K \rightarrow K^{\prime}, f \times F$ denotes their product, the map $f \times F: N \times K \rightarrow N^{\prime} \times K^{\prime}$ defined by $f \times F\left(x_{1}, x_{2}\right)=\left(f\left(x_{1}\right), F\left(x_{2}\right)\right)$ for $x_{1} \in N$, $x_{2} \in K$.

We use the Sobolev spaces $L_{k}^{p}(M \times[\alpha, \beta])$ with inhomogeneous weights in variables on a compact product Riemannian manifold $M \times[\alpha, \beta]$. For each even integer $k>0$ and each real number $1<p<\infty, L_{k}^{p}(M \times[\alpha, \beta])$ is the Sobolev space of tempered distributions on $M \times[\alpha, \beta]$ whose first $k$-derivatives in the space direction $M$ and first $(k / 2)$-derivatives in the time direction $[\alpha, \beta]$ are $L^{p}$-integrable. For other real numbers $k \in \mathbf{R}$ we refer to Hamilton [3] for the precise definition. $L_{k}^{p}\left(M \times[\alpha, \beta], \mathbf{R}^{N}\right)$ denotes the $N$-fold direct sum of $L_{k}^{p}(M \times[\alpha, \beta])$ with itself.

3. Proof of Theorem 1. To prove Theorem 1 we follow the method of Hamilton [3] and modify the argument in Lemaire [4].

We first imbed $M^{\prime}$ into a suitable Euclidean $N$-space $\mathbf{R}^{N}$ with metric defined as in $[3, \S I V, 5]$ so that the composition of $U$ with the imbedding also satisfies (D). It is known in [3, $\S \mathrm{IV}, 11]$ that for a given initial condition the Dirichlet problem (D) has a local solution $U: M \times[0, \varepsilon] \rightarrow M^{\prime}, \varepsilon>0$, of class $L_{2}^{p}\left(p>\operatorname{dim} M^{\prime}+2\right)$, which is unique, and $C^{\infty}$ except on the corner $\partial M \times 0$. 
Let $U: M \times[0, \omega) \rightarrow M^{\prime}$ be a maximal solution of the Dirichlet problem (D). Our goal is to prove $\omega=\infty$. To this end, we suppose $\omega<\infty$ and will prove that all the derivatives of $U$ remain uniformly bounded on $M \times[0, \omega)$. Then $U$ extends smoothly to a solution on $M \times[0, \omega]$ and hence to that on $M \times[0, \omega+\varepsilon], \varepsilon>0$. This shows that $U$ is not maximal and $\omega$ must be infinity in consequence.

In what follows, we denote various constants independent of $t \in[0, \omega)$ by the same letter $C$ and covariant differentiation by $\nabla$. Set $e=e(U)$ and $E=E(U)$. Define $\kappa=\frac{1}{2}|\partial U / \partial t|^{2}$ and $H=\int_{M} \kappa d \mu_{M}$. Then, since the energy density $e(F)$ of $F$ is assumed to be constant and the sectional curvature of $N^{\prime}$ nonpositive, we have

LEMMA 1 [4]. (i) For all $t \in[0, \omega)$ the solution $U$ is of the form $U=f_{t} \times F$ with $f_{t}$ : $N \times t \rightarrow N^{\prime}$.

(ii) $\partial e / \partial t<\Delta e+C_{1} e+C_{1}-|\nabla \nabla U|^{2}$ on $M \times[0, \omega)$.

(iii) $\partial \kappa / \partial t \leqslant \Delta \kappa+C_{2} \kappa-|\nabla \partial U / \partial t|^{2}$ on $M \times[0, \omega)$.

Let $\nu$ be the outward unit normal vector of $\partial M$ in $M$ and $\partial / \partial \nu$ denote the normal derivative. Then from the Dirichlet boundary condition we have

LeMMA $2[3, \S \mathrm{V}, 3,4]$. (i) $d E / d t=-2 H$ for all $t \in[0, \omega)$.

(ii) $\partial e / \partial \nu=2 m e+a \nabla \nabla U+b \nabla U$ on $\partial M \times[0, \omega)$, where $a \nabla \nabla U+b \nabla U$ is $a$ second order linear operator with $C^{\infty}$ coefficients depending only on $U \mid \partial M \times[0, \omega)$ $=u$ and $m$ is the mean curvature of $\partial M$ in $M$.

(iii) $\kappa=0$ and $\partial \kappa / \partial \nu=0$ on $\partial M \times[0, \omega)$.

Set $\Theta=\kappa \exp \left(-C_{2} t\right)$ and $T=\int_{M} \Theta d \mu_{M}=H \exp \left(-C_{2} t\right)$. Then $\partial \Theta / \partial t<\Delta \Theta$ by Lemma 1(iii) and $\partial \Theta / \partial \nu=0$ by Lemma 2(iii). Using Green's theorem

$$
\frac{\partial T}{\partial t}=\int_{M} \frac{\partial \Theta}{\partial t} d \mu_{M} \leqslant \int_{M} \Delta \Theta d \mu_{M}=\int_{\partial M} \frac{\partial \Theta}{\partial \nu} d \mu_{\partial M}=0 .
$$

Thus $T$ is monotone decreasing and hence $T<T_{0}$, that is $H<H_{0} \exp \left(C_{2} t\right)$. Since $d E / d t=-2 H<0$ by Lemma 2(i), $E$ is monotone decreasing, $E<E_{0}$ and $-d E / d t$ $<2 \mathrm{H}_{0} \exp \left(C_{2} t\right)$. From Lemma 1(ii) we have

$$
\frac{d E}{d t}=\int_{M} \frac{\partial e}{\partial t} d \mu_{M} \leqslant \int_{M} \Delta e d \mu_{M}-\int_{M}|\nabla \nabla U|^{2} d \mu_{M}+C,
$$

and from Lemma 2(ii)

$$
\int_{M} \Delta e d \mu_{M}=\int_{\partial M} \frac{\partial e}{\partial \nu} d \mu_{\partial M} \leqslant C\left\{\int_{\partial M}|\nabla U|^{2} d \mu_{\partial M}+\int_{\partial M}|\nabla \nabla U| d \mu_{\partial M}+1\right\} .
$$

It follows from these that

$$
\int_{M}|\nabla \nabla U|^{2} d \mu_{M} \leqslant C\left\{\int_{\partial M}|\nabla U|^{2} d \mu_{\partial M}+\int_{\partial M}|\nabla \nabla U| d \mu_{\partial M}+\exp (C t)\right\} .
$$

Now fix a $\delta>0$ with $0<\delta<\omega / 4$. We will estimate the Sobolev norms of $U$ on $M \times[t, t+\delta](\delta \leqslant t<\omega-\delta)$. We write simply $\|U\|_{L_{k}(M \times[t, t+\delta \mathrm{D}}$ for $\|U\|_{L R\left(M \times[t, t+\delta], \mathbf{R}^{N}\right)}$. 
$L_{2}^{2}$ norm. Recall that Image $U$, the image of $U$ in $\mathbf{R}^{N}$, is bounded, $E<E_{0}$ and $H<H_{0} \exp \left(C_{2} t\right)$. Then we get from (1) that

$$
\begin{aligned}
\|U\|_{L_{2}^{2}(M \times[t, t+\delta])}^{2} & =\int_{M \times[t, t+\delta]}\left(|U|^{2}+|\nabla U|^{2}+|\nabla \nabla U|^{2}+\left|\frac{\partial U}{\partial t}\right|^{2}\right) d \mu_{M} d t \\
& <C \int_{\partial M \times[t, t+\delta]}\left(|\nabla U|^{2}+|\nabla \nabla U|\right) d \mu_{\partial M} d t+C \exp (C t) .
\end{aligned}
$$

It is known in $[3, \S \mathrm{V}, 6,8]$ that

$$
\begin{gathered}
\int_{\partial M \times[t, t+\delta]}|\nabla U|^{2} d \mu_{\partial M} d t<C\|U\|_{L_{2}^{2}(M \times[t, t+\delta])}^{4 / 3}, \\
\int_{\partial M \times[t, t+\delta]}|\nabla \nabla U| d \mu_{\partial M} d t<C\left(1+\|U\|_{L_{2}^{2}(M \times[t-\delta, t+\delta])}\right)^{3 / 2} .
\end{gathered}
$$

From (2) and (3) it then follows, as in the proof of the theorem in $[3, \S I V, 8]$, that

$$
\|U\|_{L_{2}^{2}(M \times[t, t+\delta])}<C \exp (C t) \text {. }
$$

$L_{2}^{p}$ norm. Set $\Lambda=e+1$. Then, by Lemmas 1(ii) and 2(ii), $\Lambda$ satisfies the inequalities

$$
\begin{array}{ll}
\partial \Lambda / \partial t \leqslant \Delta \Lambda+C_{1} \Lambda & \text { on } M \times[0, \omega), \\
\partial \Lambda / \partial \nu<2 m \Lambda+a \nabla \nabla U+b \nabla U-2 m & \text { on } \partial M \times[0, \omega) .
\end{array}
$$

We define an auxiliary function $\lambda_{\theta}$ as the solution of the following linear parabolic initial-boundary value problem:

$$
\begin{aligned}
\frac{\partial \lambda_{\theta}}{\partial t} & =\Delta \lambda_{\theta}+C_{1} \lambda_{\theta} & & \text { on } M \times[\theta-\delta, \theta+\delta], \\
\lambda_{\theta} & =\Lambda & & \text { on } M \times\{\theta-\delta\}, \\
\frac{\partial \lambda_{\theta}}{\partial \nu} & =2 m \lambda_{\theta}+a \nabla \nabla U+b \nabla U-2 m & & \text { on } \partial M \times[\theta-\delta, \theta+\delta] .
\end{aligned}
$$

The problem (5) has a unique classical solution on $M \times[\theta-\delta, \theta+\delta][3, \S I V, 8]$. It follows from the maximum principle $[3, \S \mathrm{V}, 9]$ applied to $\Lambda-\lambda_{\theta}$ that $\Lambda<\lambda_{\theta}$ on $M \times[\theta-\delta, \theta+\delta]$.

By the $L^{1}$ form of the maximum principle [3, $\left.\S \mathrm{V}, 9\right]$ applied to (5) we have

$$
\begin{aligned}
& \left\|\lambda_{\theta}\right\|_{L^{\prime}(M \times[\theta-\delta, \theta+\delta])} \\
& \quad<C\left(\|\Lambda\|_{L^{\prime}(M \times\{\theta-\delta\})}+\|a \nabla \nabla U+b \nabla U-2 m\|_{L^{1}(\partial M \times[\theta-\delta, \theta+\delta])}\right),
\end{aligned}
$$

from which we get

$$
\left\|\lambda_{\theta}\right\|_{L^{1}(M \times[\theta-\delta, \theta+\delta])}<C \exp (C \theta)
$$

due to (3) and (4). On the other hand, by the dual version of the Sobolev imbedding theorem $[3, \S \mathrm{V}, 9]$ we have, for $k>p+2,\left\|\lambda_{\theta}\right\|_{L_{-k}(M \times[\theta-\delta, \theta+\delta D}<$ $C\left\|\lambda_{\theta}\right\|_{L^{\prime}(M \times[\theta-\delta, \theta+\delta D}$. Hence for $k>p+2,\left\|\lambda_{\theta}\right\|_{L_{-k}^{p}(M \times[\theta-\delta, \theta+\delta D}<C \exp (C \theta)$. Then, by a mimicry of the proof of the theorem in $[3, \S \mathrm{V}, 11]$ it is verified that

$$
\|U\|_{L_{2}(M \times[t, t+\delta])}<C \exp (C t) \text {. }
$$


$L_{k}^{p}$ norm. The estimate for the $L_{k}^{p}$ norm $\|U\|_{L_{k}}$ can be obtained from (4) and (6) by induction on real values of $k$ in increments less than 1 . The proof proceeds as in $[3, \S \mathrm{V}, 12]$ and we have $\|U\|_{L_{k}(M \times[t, t+\delta])} \leqslant C \exp (C t)$.

In consequence, all the $L_{k}^{p}$ norms of $U$ remain bounded on $M \times[0, \omega)$, since the constants $C$ are independent of $t \in[0, \omega) ; C$ may depend on $\delta$, but this does not concern us, for $\delta$ is fixed once and for all. Hence, by the Sobolev imbedding theorem [3, §II, 13], all the derivatives $(\partial / \partial t)^{r} \nabla^{s} U$ of $U$ are uniformly bounded on $M \times[0, \omega)$. This completes the proof.

4. Remarks. The reason for assuming the boundary $\partial N^{\prime}$ of $N^{\prime}$ to be convex in Theorem 1 can be found in $[3, \S I V, 3]$. Theorem 1 remains valid even when $N^{\prime}$ is without boundary, $\partial N^{\prime}=\varnothing$.

There are several examples of harmonic maps $F: K \rightarrow K^{\prime}, \tau(F)=0$, with constant energy density $e(F)$. For instance: the identity maps when $K=K^{\prime}$, minimal immersions of Riemannian manifolds and the Hopf fibrations $S^{3} \rightarrow S^{2}$, $S^{7} \rightarrow S^{4}, S^{15} \rightarrow S^{8}$ in their classical polynomial representations. See [2].

By slight modifications of the proof of Theorem 1, we can prove a similar result for the Neumann problem. In fact, we have

TheOREM 2. Let $M=N \times_{\varphi} K, M^{\prime}=N^{\prime} \times_{\psi} K^{\prime}$ and $F: K \rightarrow K^{\prime}$ be as in Theorem 1. Let $f_{0}: N \rightarrow N^{\prime}$ be a $C^{\infty}$ map with $\partial f_{0} / \partial \nu=0$ on $\partial N$. Then the Neumann initial-boundary value problem for the nonlinear parabolic equation of Eells-Sampson

$$
\begin{array}{ll}
\partial U / \partial t=\tau(U) & \text { on } M \times[0, \infty), \\
U=f_{0} \times F & \text { on } M \times 0, \\
\partial U / \partial \nu=0 & \text { on } \partial M \times[0, \infty)
\end{array}
$$

has a global solution $U: M \times[0, \infty) \rightarrow M^{\prime}$, which is unique, and $C^{\infty}$ except on the corner $\partial M \times 0$.

We refer to $[3, \S \mathrm{V}, 14]$ for an indication of necessary modifications of the proof.

Compare Theorem 2 with Theorem 1 of [5], in which an estimate for the existence time of solutions of the Neumann problem $(N)$ is given in terms of the $L^{\infty}$ norm of initial values and the bounds of the sectional curvatures of the source and target manifolds.

\section{REFERENCES}

1. R. L. Bishop and B. O’Neill, Manifolds of negative curvature, Trans. Amer. Math. Soc. 145 (1969), $1-49$.

2. J. Eells and J. H. Sampson, Harmonic mappings of Riemannian manifolds, Amer. J. Math. 86 (1964), 109-160.

3. R. S. Hamilton, Harmonic maps of manifolds with boundary, Lecture Notes in Math., vol. 471, Springer-Verlag, Berlin and New York, 1975.

4. L. Lemaire, Applications harmonique de variétés produits, Comment. Math. Helv. 52 (1977), 11-24.

5. S. Nishikawa, On the Neumann problem for the nonlinear parabolic equation of Eells-Sampson and harmonic mappings, Math. Ann. 249 (1980), 177-190.

Department of Mathematics, College of General Education, Nagoya Universtty, Nagoya 464, JAPAN 